\title{
Perfil metabólico e ponderal de leitões submetidos a dietas com diferentes teores de fósforo em ambientes térmicos distintos
}

\author{
Waleska de Melo Ferreira Dantas(1), José Dantas Ribeiro Filho(1), José Domingos Guimarães ${ }^{(1)}$ \\ e Simone Eliza Facioni Guimarães ${ }^{(2)}$
}

\begin{abstract}
(1)Universidade Federal de Viçosa (UFV), Departamento de Veterinária, Avenida Peter Henry Rolfs, s/no, Campus Universitário, CEP $36570-000$ Viçosa, MG, Brasil. E-mail: wafedantas@yahoo.com.br, dantas@ufv.br, jdguima@ufv.br(2)UFV, Departamento de Zootecnia, Avenida Peter Henry Rolfs, s/no, Campus Universitário, CEP 36570-000 Viçosa, MG, Brasil. E-mail: sfacioni@ufv.br
\end{abstract}

\begin{abstract}
Resumo - O objetivo deste trabalho foi avaliar o efeito de dietas com diferentes concentrações de fósforo disponível sobre o perfil metabólico e o peso corporal de leitões em fase inicial de crescimento, mantidos em ambientes térmicos distintos. Foram utilizados 120 leitões machos castrados, de linhagem comercial e com peso corporal médio de $15 \mathrm{~kg}$. Os tratamentos consistiram de dietas com 0,107, 0,214, 0,321, 0,428 e $0,535 \%$ de fósforo disponível, e de dois ambientes: termoneutro e quente. O peso corporal foi determinado no início e no final da fase experimental, e amostras de sangue foram coletadas para a medição de eletrólitos, albumina, proteína total, substâncias nitrogenadas, fosfatase alcalina total e óssea, hormônios tireoidianos, glicose e lactato. Tanto as dietas quanto os ambientes produziram efeito sobre o ganho de peso corporal e sobre os valores de sódio, potássio, cálcio total, magnésio, fósforo, fosfatase alcalina total e óssea, $\mathrm{T}_{4}$ livre e lactato. $\mathrm{O}$ aumento nos teores de fósforo na dieta tem efeito positivo sobre o peso corporal, nos ambientes estudados, mas desequilibra as relações sanguíneas cálcio:fósforo. O estresse por calor diminui as concentrações de glicose, fosfatase alcalina total e $\mathrm{T}_{4}$ livre no sangue, e aumenta as de lactato. Parâmetros bioquímicos sanguíneos retratam o estado metabólico dos animais e podem ser usados para avaliação do balanço nutricional e para detecção precoce de alterações metabólicas.
\end{abstract}

Termos para indexação: bioquímica clínica, estresse por calor, fosfato bicálcico, suplementação mineral.

\section{Metabolic profile and body weight of piglets fed with different levels of phosphorus in distinct thermal environments}

\begin{abstract}
The objective of this work was to evaluate the effect of diets with different available phosphorus contents on metabolic profile and on body weight of piglets at baseline, maintained in distinct thermal environments. One hundred and twenty barrow piglets from commercial strain and with an average body weight of $15 \mathrm{~kg}$ were used. The treatments consisted of diets with $0.107,0.214,0.321,0.428$, and $0.535 \%$ available phosphorus, and of two environments: thermoneutral and warm. Body weight was determined at the beginning and at the end of the experimental period, and blood samples were taken for measuring electrolytes, albumin, total protein, nitrogenous substances, total and bone alkaline phosphatase, thyroid hormones, glucose, and lactate. Both diets and environments had effect on body weight gain and on the values of sodium, potassium, total calcium, magnesium, phosphorus, total and bone alkaline phosphatase, free $\mathrm{T}_{4}$, and lactate. The increase in phosphorus contents in the diet has a positive effect on body weight in the studied environments, but it unbalances blood calcium:phosphorus relations. Heat stress decreases the levels of glucose, total alkaline phosphatase, and free $T_{4}$ in the blood, and increases those of lactate. Blood biochemical parameters reflect the metabolic state of the animals and can be used to evaluate nutritional balance and for early detection of metabolic alterations.
\end{abstract}

Index terms: clinical biochemistry, heat stress, bicalcium phosphate, mineral supplementation.

\section{Introdução}

Tanto o milho quanto a soja, ingredientes comuns nas rações para suínos, não dispõem de quantidades adequadas de fósforo disponível para atender às exigências nutricionais desses animais. Assim, a adição de fosfato bicálcico na dieta é necessária e consiste na principal fonte de fósforo inorgânico em dietas animais (Saraiva et al., 2009).

A limitação na quantidade ingerida de um único nutriente pode diminuir ou até mesmo deter o crescimento animal (Díaz González \& Silva, 2006). 
Além disso, sabe-se que a temperatura ambiente reduz de forma significativa a ingestão voluntária de ração, e o estresse por calor é considerado um dos principais fatores relacionado à queda no desempenho dos animais (Alebrante et al., 2011b).

O Brasil é um país com grandes variações de temperatura e umidade entre as regiões, e a criação de suínos tem sido considerada de maior viabilidade nas regiões Sul e Sudeste, em razão das condições climáticas favoráveis ao desempenho da espécie, que apresenta perfil homeostático de conforto entre $20 \mathrm{e}$ $24^{\circ} \mathrm{C}$, e é sensível a altas temperaturas (Coffey et al., 2000). A sensibilidade dos suínos ao calor deve-se a sua incapacidade de transpirar quando a temperatura ambiente aproxima-se da temperatura corporal. Isso ocorre porque a espécie apresenta glândulas sudoríparas afuncionais, e a forma mais eficiente para dissipar o calor corporal é o aumento da frequência respiratória (Klein, 2014).

Em decorrência do elevado custo da alimentação animal, que corresponde de 60 a $80 \%$ do custo total na produção de suínos (Talamini et al., 2006), e da grande influência que o conforto térmico tem sobre a ingestão de ração, trabalhos que abordem a interação entre esses fatores são importantes. Além disso, é relevante que se avalie o efeito da disponibilidade de fósforo na ração sobre o perfil metabólico e o desempenho animal, uma vez que os principais ingredientes das rações (milho e soja) apresentam baixos conteúdos do elemento.

O objetivo deste trabalho foi avaliar o efeito de dietas com diferentes concentrações de fósforo disponível, sobre o perfil metabólico e o peso corporal de leitões em fase inicial de crescimento, mantidos em ambientes termoneutro e quente.

\section{Material e Métodos}

O experimento foi realizado nas câmaras climáticas do setor de suinocultura do Departamento de Zootecnia, do Centro de Ciências Agrárias, da Universidade Federal de Viçosa (UFV), em Minas Gerais. O estudo foi aprovado pelo Comitê de Ética para Uso de Animais da UFV, sob protocolo no 47/2011.

Foram utilizados 120 leitões de linhagem comercial, divididos em dois grupos com 60 animais cada um. Os animais foram mantidos, no grupo 1 , em ambiente termoneutro, com temperatura em torno de $24,5 \pm 1,2^{\circ} \mathrm{C}$ e umidade relativa de $76,3 \pm 8,5 \%$; e, no grupo 2 , em ambiente quente, com temperatura de $34,1 \pm 0,8^{\circ} \mathrm{C}$ e umidade relativa de $70,1 \pm 8,1 \%$. As temperaturas foram mantidas por meio de aquecedores elétricos, modelo Seletro (Seletro, Formiga, MG), e por dois aparelhos de ar condicionado de 18.000 BTUs cada um. O ambiente térmico no local foi monitorado três vezes ao dia (7:00, 12:00 e 17:00), com uso de termômetro colocado no meio de uma gaiola vazia, à altura média do corpo dos suínos. A partir desses dados, calculou-se o índice de temperatura de globo negro e umidade (ITGU), de acordo com Buffington et al. (1981).

O peso corporal médio inicial dos leitões foi, respectivamente, de $14,97 \pm 0,36$ e $15,10 \pm 0,31 \mathrm{~kg}$, para os animais dos grupos $1\left(24^{\circ} \mathrm{C}\right)$ e $2\left(34^{\circ} \mathrm{C}\right)$. Os animais foram alojados em gaiolas metálicas suspensas $\left(2 \mathrm{~m}^{2}\right.$ por animal), com piso e laterais teladas, dotadas de comedouros semiautomáticos e bebedouros tipo chupeta, em sala climatizada, construída em alvenaria, com piso de concreto, coberta com telhas de cerâmica, forração em madeira e janelas de vidro com sistema basculante. Utilizou-se o delineamento experimental utilizado de blocos ao acaso, com cinco tratamentos (dietas), seis repetições e dois animais por unidade experimental. A unidade experimental foi representada pela gaiola, e o período experimental compreendeu 25 dias.

Os tratamentos consistiram de: uma ração basal, sem suplementação com fósforo, composta principalmente por milho e farelo de soja, suplementada com vitaminas, minerais e aminoácidos, contendo $0,107 \%$ de fósforo disponível (dieta 1); e dessa ração basal suplementada com quatro concentrações de fosfato bicálcico comercial: $0,214 \%$ (dieta 2), $0,321 \%$ (dieta 3), 0,428\% (dieta 4) e 0,535\% (dieta 5) de fósforo disponível. As dietas foram isoproteicas, isoenergéticas e isocálcicas (Tabela 1). As rações foram formuladas de acordo com as recomendações para leitões de 15 a $30 \mathrm{~kg}$, segundo Rostagno et al. (2005), exceto quanto ao fósforo. Os animais receberam as rações experimentais e água ad libitum, e foram pesados no início (M0) e no fim do período experimental (M1), para a determinação do ganho de peso corporal.

Amostras de sangue foram coletadas imediatamente antes (M0) e ao término do período experimental (M1), por meio de venopunção do sinus orbital, com agulhas descartáveis hipodérmicas 40x16, sem jejum prévio. Após as coletas, as amostras foram acondicionadas em frascos de $10 \mathrm{~mL}$ sem anticoagulante, para obtenção 
de soro, e em frascos de $5 \mathrm{~mL}$ contendo fluoreto de sódio, para obtenção do plasma. As alíquotas de soro e plasma foram, então, mantidas congeladas a $-20^{\circ} \mathrm{C}$ até o momento das análises laboratoriais, em que foram medidas as concentrações de: sódio e potássio por espectrofotômetro de chama, com uso de fotômetro de chama, modelo B462 (Micronal, S.A., São Paulo, $\mathrm{SP}$ ); cloreto, fósforo, cálcio total, magnésio, albumina, proteína total, ureia, creatinina, fosfatase alcalina total, glicose e lactato, pelo método colorimétrico, com uso de aparelho eletrônico de automação Humastar300 (Human - Artigos e Equipamentos para Laboratórios, Itabira, MG); e de TSH, $\mathrm{T}_{4}$ livre e fosfatase alcalina óssea, pelo método de quimioluminescência, com uso do aparelho Access Immunoassay System (Beckman Coulter do Brasil, Rio de Janeiro, RJ).
A estatística descritiva foi utilizada para a obtenção das médias e do desvio-padrão das variáveis estudadas. Os dados foram avaliados pelos testes de Lilliefors e Cochran \& Bartlet, para verificar a normalidade dos dados e a homogeneidade das variâncias, respectivamente. Procedeu-se à análise de variância e, quando as variáveis não atendiam às suas premissas, elas foram submetidas aos procedimentos não paramétricos, pelo teste de Kruskal-Wallis e Wilcoxon. Quando significativos, os dados foram submetidos à análise de regressão para determinação dos pontos de função máxima e mínima dos níveis de fósforo. Além disso, avaliou-se a interação entre ambientes e dietas. As análises foram feitas com uso do programa SAEG 9.0 (SAEG, 2007) e o PROC REG (SAS Institute, 2000). Em todas as análises, a significância adotada foi de 5\%.

Tabela 1. Composição percentual e calculada das rações experimentais.

\begin{tabular}{|c|c|c|c|c|c|}
\hline \multirow[t]{2}{*}{ Ingredientes } & \multicolumn{5}{|c|}{ Nível de fósforo disponível nas dietas (\%) } \\
\hline & 0,107 & 0,214 & 0,321 & 0,428 & 0,535 \\
\hline Milho de grão moído & 62,011 & 62,011 & 62,011 & 62,011 & 62,011 \\
\hline Farelo de soja $45 / 80$ & 32,000 & 32,000 & 32,000 & 32,000 & 32,000 \\
\hline Óleo de soja & 1,810 & 1,810 & 1,810 & 1,810 & 1,810 \\
\hline Fosfato bicálcico & 0,000 & 0,577 & 1,156 & 1,734 & 2,312 \\
\hline Calcário & 1,837 & 1,467 & 1,098 & 0,730 & 0,360 \\
\hline Inerte & 1,200 & 0,993 & 0,783 & 0,573 & 0,365 \\
\hline Sal comum & 0,456 & 0,456 & 0,456 & 0,456 & 0,456 \\
\hline Premix - vitaminas ${ }^{(1)}$ & 0,100 & 0,100 & 0,100 & 0,100 & 0,100 \\
\hline Premix - minerais ${ }^{(2)}$ & 0,050 & 0,050 & 0,050 & 0,050 & 0,050 \\
\hline Sulfato de colistina ${ }^{(3)}$ & 0,075 & 0,075 & 0,075 & 0,075 & 0,075 \\
\hline Fosfato de tilosina ${ }^{(4)}$ & 0,030 & 0,030 & 0,030 & 0,030 & 0,030 \\
\hline L-lisina $\mathrm{HCl}$ & 0,282 & 0,282 & 0,282 & 0,282 & 0,282 \\
\hline DL-metionina & 0,073 & 0,073 & 0,073 & 0,073 & 0,073 \\
\hline L-treonina & 0,066 & 0,066 & 0,066 & 0,066 & 0,066 \\
\hline BHT & 0,010 & 0,010 & 0,010 & 0,010 & 0,010 \\
\hline Total & 100,00 & 100,00 & 100,00 & 100,00 & 100,00 \\
\hline \multicolumn{6}{|l|}{ Composição calculada } \\
\hline Energia metabolizável $\left(\mathrm{kcal} \mathrm{kg}^{-1}\right)$ & 3.250 & 3.250 & 3.250 & 3.250 & 3.250 \\
\hline Proteína bruta $(\%)$ & 19,981 & 19,981 & 19,981 & 19,981 & 19,981 \\
\hline Lisina digestível (\%) & 1,146 & 1,146 & 1,146 & 1,146 & 1,146 \\
\hline Metionina + cistina digestível (\%) & 0,641 & 0,641 & 0,641 & 0,641 & 0,641 \\
\hline Treonina digestível (\%) & 0,721 & 0,721 & 0,721 & 0,721 & 0,721 \\
\hline Sódio $(\%)$ & 0,200 & 0,200 & 0,200 & 0,200 & 0,200 \\
\hline Cálcio (\%) & 0,800 & 0,800 & 0,800 & 0,800 & 0,800 \\
\hline P total $(\%)$ & 0,319 & 0,425 & 0,532 & 0,639 & 0,746 \\
\hline P disponível (\%) & 0,107 & 0,214 & 0,321 & 0,428 & 0,535 \\
\hline Relação Ca:P & $2,5: 1$ & $1,9: 1$ & $1,5: 1$ & $1,25: 1$ & $1,1: 1$ \\
\hline
\end{tabular}

(1) Conteúdo por kg de produto: 8.000.000 UI de vitamina A; 2.000.000 UI de vitamina D3; $10.000 \mathrm{mg}$ de vitamina E; $800 \mathrm{mg}$ de ácido fólico; $12.000 \mathrm{mg}$ de pantotenato de cálcio; $50 \mathrm{mg}$ de biotina; $25.000 \mathrm{mg}$ de niacina; $2.000 \mathrm{mg}$ de piridoxina; $5.000 \mathrm{mg}$ de riboflavina; $1.500 \mathrm{mg}$ de tiamina; $20.000 \mathrm{mg}$ de vitamina B12; $1.500 \mathrm{mg}$ de vitamina K3; $320 \mathrm{mg}$ de selênio; e $30.000 \mathrm{mg}$ de antioxidante. ${ }^{(2)}$ Conteúdo por kg de produto: $30.000 \mathrm{mg}$ de cobre; $160.000 \mathrm{mg}$ de zinco; $1.900 \mathrm{mg}$ de iodo; $100.000 \mathrm{mg}$ de ferro; e $70.000 \mathrm{mg}$ de manganês. ${ }^{(3)}$ Conteúdo por kg de produto: $80.000 \mathrm{mg}$ de colistina. ${ }^{(4)}$ Conteúdo por kg de produto: $400.000 \mathrm{mg}$ de tilosina. 


\section{Resultados e Discussão}

Durante todo o período experimental, o índice ITGU foi de $86,7 \pm 1,14$ para os animais do grupo 2 . Portanto, pode-se inferir que os animais desse grupo foram expostos a estresse térmico, o que corrobora a informação de que o ambiente termoneutro para leitões em fase inicial de crescimento varia entre 18 e $28^{\circ} \mathrm{C}$ (Coffey et al., 2000), e de que ITGU acima de 80,0 caracteriza ambiente de estresse por calor (Campos et al., 2008).

O peso corporal dos animais do grupo 1 foi significativamente influenciado pela suplementação de fosfato bicálcico, tendo apresentado comportamento cúbico (Figura $1 \mathrm{~A}$ ). Esses resultados confirmam os de Saraiva et al. (2009), que verificaram melhores ganhos com concentrações de fósforo disponível entre 0,509 e $0,477 \%$, e os de Alebrante et al. (2011a), que constataram melhores resultados com as concentrações de 0,443, 0,461 e 0,525\%. Dantas et al. (2010), em estudo com suínos em fase de terminação $(60-90 \mathrm{~kg})$, observaram melhor ganho de peso com suplementação de $0,330 \%$ de fósforo disponível. Nesses trabalhos, os leitões foram mantidos em ambiente de conforto térmico.

No grupo 2, observou-se comportamento quadrático (Figura $1 \mathrm{~B}$ ), em que o maior peso corporal foi obtido com a dieta $4(0,428 \%)$. Resultados similares foram relatados por Alebrante et al. (2011b), que trabalharam com suínos na mesma faixa etária que os do presente
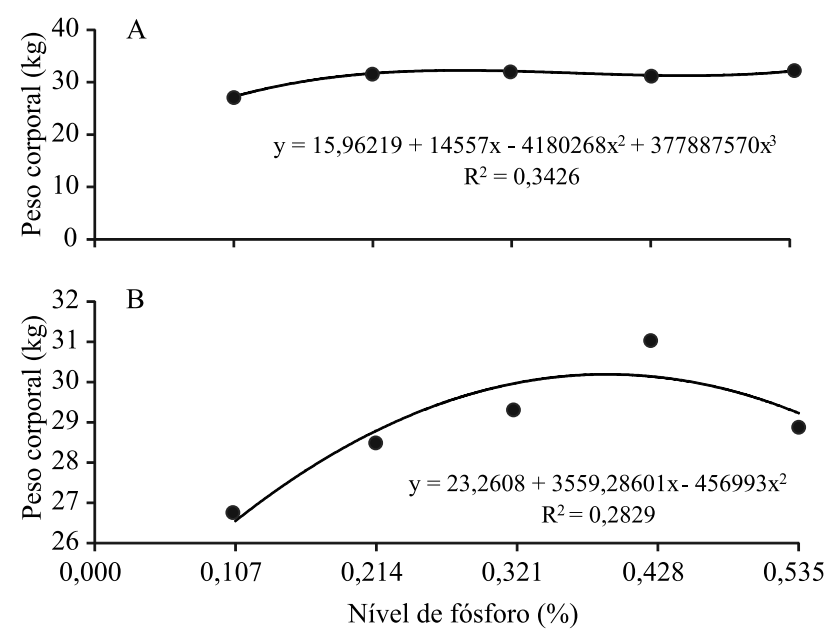

Figura 1. Comportamento do peso corporal de leitões em fase inicial de crescimento $(15-30 \mathrm{~kg})$, nos grupos 1 (A) e 2 (B), em função de diferentes níveis de fósforo na dieta. experimento e com o mesmo ambiente térmico $\left(34^{\circ} \mathrm{C}\right)$. Os resultados obtidos são indicativos de que as concentrações de fósforo recomendadas $(0,107 \%$ de fósforo disponível) necessitam de ajustes, para não limitar o novo potencial genético dos animais, principalmente em ambientes quentes, nos quais o animal reduz seu consumo alimentar na tentativa de diminuir a produção de calor.

O peso corporal final entre os ambientes estudados (grupos 1 e 2) variou de acordo com as dietas, e o maior valor foi registrado no ambiente termoneutro. Esse resultado confirma a sensibilidade dos suínos a altas temperaturas (Coffey et al., 2000), por terem dificuldade em dissipar o calor (Klein, 2014). Conforme Manno et al. (2005), altas temperaturas têm influência negativa na conversão alimentar e na deposição de proteína na carcaça, além de aumentarem a frequência respiratória e a temperatura retal dos suínos. A suplementação de fósforo foi de fundamental importância para que os animais mantidos em ambiente quente $\left(34^{\circ} \mathrm{C}\right)$ pudessem ter o mesmo desempenho que os mantidos em ambiente termoneutro $\left(24^{\circ} \mathrm{C}\right)$. Esse resultado é indicativo de que o teor de fosfato bicálcico na dieta de $0,428 \%$ de fósforo disponível atingiu o ponto de equilíbrio no organismo, ou seja, supriu a energia requerida para a mantença e a necessária para o metabolismo proteico utilizada na formação muscular, embora a temperatura ambiente de $34^{\circ} \mathrm{C}$ não tenha sido adequada.

As diferentes dietas, dentro de cada grupo, não diferiram quanto aos valores séricos de cloreto, sódio, potássio e magnésio (Tabela 2). Porém, houve diferença entre os grupos 1 e 2 nas concentrações de sódio, potássio e magnésio. O sódio apresentou comportamento linear no grupo 2 e quadrático no grupo 1, e exibiu comportamento semelhante ao do potássio, com valores maiores na dieta $3(0,321 \%)$ do grupo 2. Apesar de as concentrações de sódio e potássio séricos terem sofrido influência da dieta, seus valores permaneceram na faixa de referência para a espécie, propostos por Kaneko et al. (2008) e Radostits et al. (2010).

As dietas, dentro dos grupos, também não diferiram quanto aos valores da diferença entre íons fortes (DIF); contudo, na comparação entre os grupos, eles diferiram na dieta 3 . Essa diferença foi decorrente da influência dos valores séricos de sódio e potássio, que aumentaram no ambiente quente (Tabela 2). 
Esse parâmetro foi determinado pela fórmula $\mathrm{DIF}=\left[\mathrm{Na}^{+}\right]+\left[\mathrm{K}^{+}\right]-\left[\mathrm{Cl}^{-}\right]$(Constable, 2003). Como não há valores de referência para essa variável na literatura e como as variáveis responsáveis por sua determinação se mantiveram na faixa de referência, acredita-se que os animais não apresentaram acidose ou alcalose metabólica ocasionada por desequilíbrio de íons fortes.

Embora tenha sido observada diferença nas concentrações séricas de magnésio, na comparação entre ambientes, seus valores permaneceram dentro dos limites de referência (Kaneko et al., 2008; Radostits et al., 2010). Isso implica que, provavelmente, a temperatura ambiente não influenciou a excreção renal.

O cálcio total e o fósforo diferiram entre as dietas, em cada ambiente, e entre os ambientes. $O$ cálcio total apresentou comportamento linear no grupo 2 e quártico no grupo 1 (Figura 2). Os maiores valores foram obtidos nas dietas 1 e 2, nos grupos 1 e 2, respectivamente. Já o fósforo apresentou comportamento quadrático nos dois grupos estudados. Suas maiores concentrações foram verificadas nas dietas 3, 4 e 5, em ambos os grupos. É interessante ressaltar que, no ambiente quente $\left(34^{\circ} \mathrm{C}\right)$, as concentrações sanguíneas de fósforo foram maiores que as observadas no ambiente termoneutro $\left(24^{\circ} \mathrm{C}\right)$, exceto na dieta 4 , em que não houve diferença entre os grupos.

À medida que a suplementação de fósforo na dieta é aumentada, a relação cálcio:fósforo no sangue se desequilibra. No presente trabalho, foram verificadas as proporções de 2,4:1, 1,6:1, 1,1:1, 1:1 e $1: 1$, respectivamente, às dietas de 1 a 5 , no ambiente termoneutro; e de $1,9: 1,1,4: 1,1,1: 1,1: 1$ e $1: 1$ no

Tabela 2. Peso corporal, concentrações séricas de sódio, potássio, cloreto, magnésio, fósforo, cálcio total, relação cálcio:fósforo e diferença de íons fortes (DIF), em leitões em fase inicial de crescimento (15-30 kg), alimentados com dietas com diferentes suplementações de fósforo disponível e mantidos em ambiente termoneutro (grupo $1,24^{\circ} \mathrm{C}$ ) ou quente (grupo $\left.2,34^{\circ} \mathrm{C}\right)^{(1)}$.

\begin{tabular}{|c|c|c|c|c|c|}
\hline \multirow[t]{2}{*}{ Grupo } & \multicolumn{5}{|c|}{ Nível de fósforo disponível nas dietas (\%) } \\
\hline & 0,107 & 0,214 & 0,321 & 0,428 & 0,535 \\
\hline & \multicolumn{5}{|c|}{ Peso (kg) } \\
\hline 1 & $27,3 \pm 1,8 \mathrm{Ab}$ & $31,3 \pm 2,4 \mathrm{Aa}$ & $32,7 \pm 2,3 \mathrm{Aa}$ & $30,9 \pm 3,9 \mathrm{Aa}$ & $32,2 \pm 2,7 \mathrm{Aa}$ \\
\hline \multirow[t]{2}{*}{2} & $26,8 \pm 2,5 \mathrm{Ac}$ & $28,5 \pm 2,3 \mathrm{Bbc}$ & $29,4 \pm 2,1 \mathrm{Bab}$ & $31,1 \pm 1,6 \mathrm{Aa}$ & $28,8 \pm 1,9 \mathrm{Babc}$ \\
\hline & \multicolumn{5}{|c|}{ Sódio (meq L-1) } \\
\hline 1 & $146,1 \pm 6,3 \mathrm{Aa}$ & $144,8 \pm 6,6$ Aa & $140,1 \pm 9,6 \mathrm{Ba}$ & $143,7 \pm 3,6 \mathrm{Aa}$ & $149,5 \pm 13,2 \mathrm{Aa}$ \\
\hline \multirow[t]{2}{*}{2} & $150,8 \pm 8,8 \mathrm{Aa}$ & $146,8 \pm 12 \mathrm{Aa}$ & $149,8 \pm 9,6 \mathrm{Aa}$ & $143,6 \pm 8,2 \mathrm{Aa}$ & $142,3 \pm 6,2 \mathrm{Aa}$ \\
\hline & \multicolumn{5}{|c|}{ Potássio (meq L $\left.{ }^{-1}\right)$} \\
\hline 1 & $6,4 \pm 1,1 \mathrm{Aa}$ & $6,1 \pm 0,8 \mathrm{Aa}$ & $6 \pm 0,5 \mathrm{Ba}$ & $6,1 \pm 0,6 \mathrm{Aa}$ & $6,4 \pm 0,5 \mathrm{Aa}$ \\
\hline \multirow[t]{2}{*}{2} & $6,4 \pm 0,7 \mathrm{Aa}$ & $6,2 \pm 0,6 \mathrm{Aa}$ & $6,5 \pm 0,5 \mathrm{Aa}$ & $6,3 \pm 0,5 \mathrm{Aa}$ & $6,5 \pm 0,8 \mathrm{Aa}$ \\
\hline & \multicolumn{5}{|c|}{ Cloreto (meq L-1) } \\
\hline 1 & $111,6 \pm 3,2 \mathrm{Aa}$ & $111,9 \pm 1,6 \mathrm{Aa}$ & $111,3 \pm 3 \mathrm{Aa}$ & $112 \pm 2,4 \mathrm{Aa}$ & $113,5 \pm 1,6 \mathrm{Aa}$ \\
\hline \multirow[t]{2}{*}{2} & $111,2 \pm 2,6 \mathrm{Aa}$ & $111,6 \pm 2,5 \mathrm{Aa}$ & $112,4 \pm 2,1 \mathrm{Aa}$ & $111,7 \pm 1,9 \mathrm{Aa}$ & $112,3 \pm 1,8 \mathrm{Aa}$ \\
\hline & \multicolumn{5}{|c|}{ Magnésio (meq L-1) } \\
\hline 1 & $2,7 \pm 0,1 \mathrm{Aa}$ & $2,7 \pm 0,2 \mathrm{Aa}$ & $2,5 \pm 0,7 \mathrm{Aa}$ & $2,7 \pm 0,1 \mathrm{Aa}$ & $2,8 \pm 0,1 \mathrm{Aa}$ \\
\hline \multirow[t]{2}{*}{2} & $2,6 \pm 0,3 \mathrm{Ba}$ & $2,6 \pm 0,3 \mathrm{Aa}$ & $2,5 \pm 0,2 \mathrm{Aa}$ & $2,6 \pm 0,2 \mathrm{Aa}$ & $2,7 \pm 0,3 \mathrm{Aa}$ \\
\hline & \multicolumn{5}{|c|}{ Fósforo (meq L $\left.{ }^{-1}\right)$} \\
\hline 1 & $5,4 \pm 0,7 \mathrm{Bc}$ & $8,2 \pm 0,9 \mathrm{Ab}$ & $10,4 \pm 1 \mathrm{Aa}$ & $10,6 \pm 0,9 \mathrm{Aa}$ & $11,3 \pm 0,8 \mathrm{Aa}$ \\
\hline \multirow[t]{2}{*}{2} & $6,4 \pm 0,9 \mathrm{Ad}$ & $8,3 \pm 1 \mathrm{Ac}$ & $9,6 \pm 0,9 \mathrm{Bb}$ & $10,9 \pm 0,6 \mathrm{Aa}$ & $10,5 \pm 0,6 \mathrm{Bab}$ \\
\hline & \multicolumn{5}{|c|}{ Cálcio (meq L-1) } \\
\hline 1 & $13,2 \pm 1 \mathrm{Aa}$ & $12,9 \pm 0,7 \mathrm{Aa}$ & $11,1 \pm 0,7 \mathrm{Ab}$ & $10,7 \pm 0,5 \mathrm{Ab}$ & $10,8 \pm 0,6 \mathrm{Ab}$ \\
\hline \multirow[t]{2}{*}{2} & $12 \pm 0,9 \mathrm{Ba}$ & $11,5 \pm 1,1 \mathrm{Ba}$ & $10,4 \pm 0,5 \mathrm{Bb}$ & $10,2 \pm 0,8 \mathrm{Ab}$ & $10 \pm 0,9 \mathrm{Bb}$ \\
\hline & \multicolumn{5}{|c|}{ Relação Ca:P } \\
\hline 1 & $2,4: 1$ & $1,6: 1$ & $1,1: 1$ & $1: 1$ & $1: 1$ \\
\hline \multirow[t]{2}{*}{2} & $1,9: 1$ & $1,4: 1$ & $1,1: 1$ & $1: 1$ & $1: 1$ \\
\hline & \multicolumn{5}{|c|}{ DIF (meq L-1) } \\
\hline 1 & $40,9 \pm 7,9 \mathrm{Aa}$ & $39,1 \pm 7,4 \mathrm{Aa}$ & $33,9 \pm 11,9 \mathrm{Ba}$ & $37,8 \pm 5,2 \mathrm{Aa}$ & $42,4 \pm 14,3 \mathrm{Aa}$ \\
\hline 2 & $46 \pm 10,3 \mathrm{Aa}$ & $41,4 \pm 12,3 \mathrm{Aa}$ & $43,8 \pm 10,2 \mathrm{Aa}$ & $38,3 \pm 8,9 \mathrm{Aa}$ & $36,5 \pm 6,3 \mathrm{Aa}$ \\
\hline
\end{tabular}


ambiente quente. Esses resultados acompanharam a relação proposta pelas dietas (Tabela 2), o que mostra a proporcionalidade entre ingestão de fósforo e seus valores sanguíneos. O organismo consegue compensar o excesso de fosfato na dieta até a relação de 1,6:1 (grupo 1) e de 1,4:1 (grupo 2), que corresponderam à dieta 3 dos dois grupos. Essa compensação é feita pela eliminação renal. Segundo Kaneko et al. (2008) e Radostits et al. (2010), a relação entre as concentrações
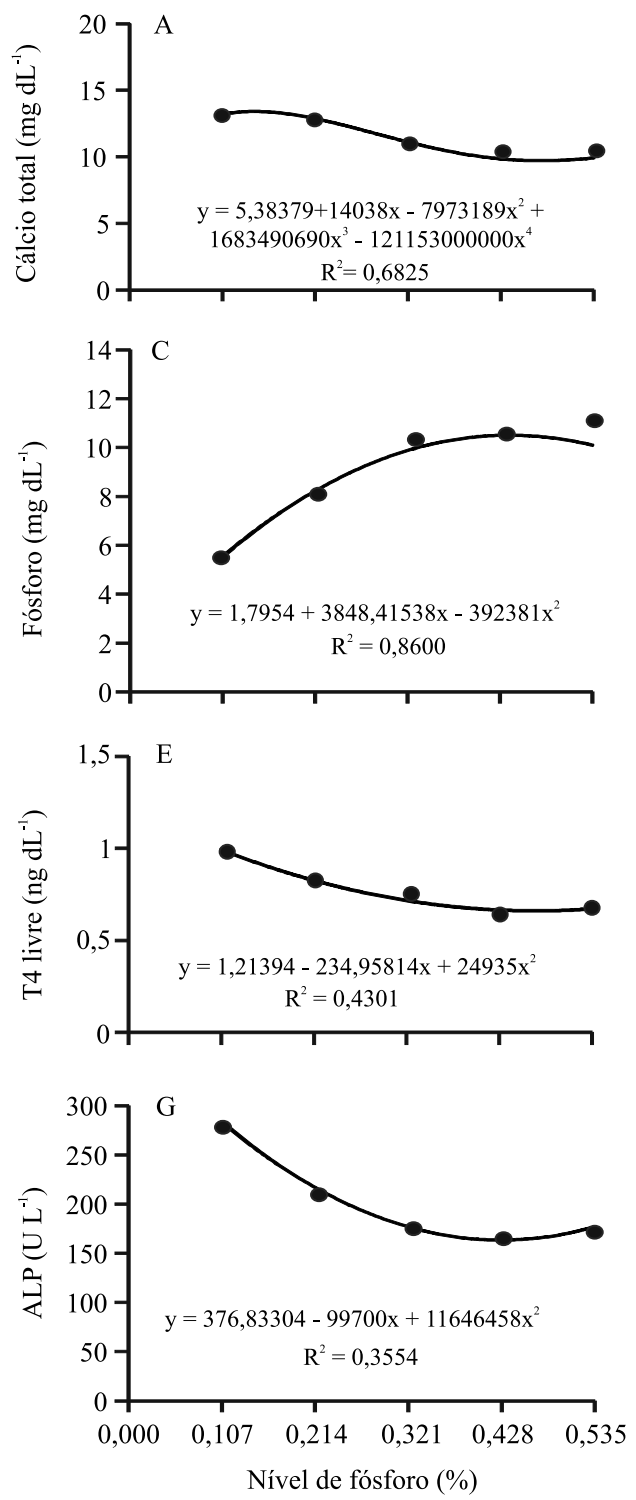

Figura 2. Concentrações séricas nos grupos 1 e 2 de leitões em fase inicial de crescimento (15-30 kg), em função de diferentes níveis de fósforo na dieta: A e B, cálcio total; C e D, fósforo; $\mathrm{E}$ e $\mathrm{F}, \mathrm{T}_{4}$ livre; e $\mathrm{G}$ e $\mathrm{H}$, fosfatase alcalina (ALP), respectivamente para os ambientes termoneutro e quente. séricas de cálcio e fósforo dentro da faixa de normalidade se encontram em torno de 1,3:1, diferente da relação constatada no presente trabalho.

As concentrações séricas de albumina, proteína total e ureia não sofreram efeitos das dietas e nem dos grupos (Tabela 3). Já a creatinina apresentou comportamento quadrático no grupo 2. No entanto, seus valores estiveram dentro da faixa de referência (Kaneko et al., 2008; Radostits et al., 2010), indício
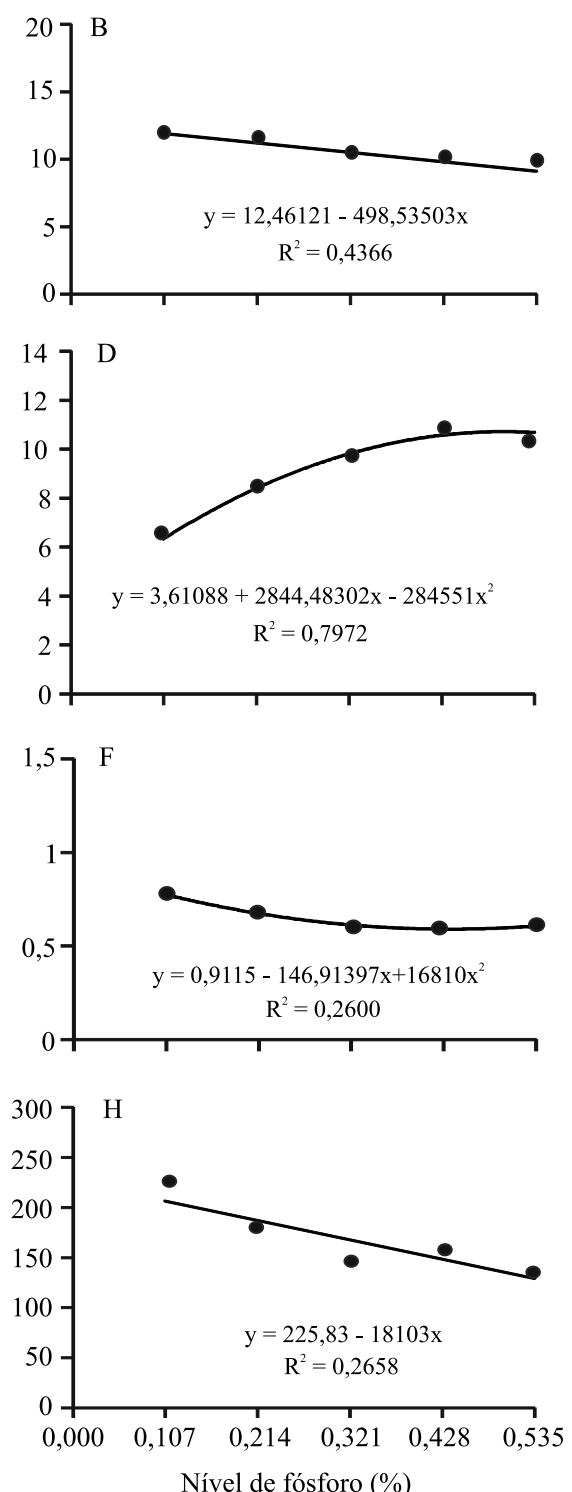
de que os rins estavam desempenhando sua função de manutenção do equilíbrio eletrolítico e do ácido base, apesar do excesso de eletrólitos presentes no sangue.

As concentrações plasmáticas de glicose diferiram entre as dietas (Tabela 3). Contudo, observou-se comportamento linear no grupo 2 , e os menores valores foram encontrados nesse ambiente. Provavelmente, a redução do consumo de alimento nos animais do grupo 2 foi a responsável pelo resultado. Estudos anteriores (Kiefer et al., 2010; Brêtas et al., 2011) mostraram que animais submetidos a ambientes quentes reduzem seu consumo voluntário de alimento. Possivelmente, a energia que seria metabolizada para a produção de carne é desviada para a energia de mantença, o que pode ter efeito mais drástico nas dietas com menores suplementações de fósforo. Porém, os valores de glicose permaneceram na faixa de referência (Kaneko et al., 2008; Radostits et al., 2010).

$\mathrm{Na}$ avaliação do lactato plasmático, o valor obtido antes dos tratamentos foi de $35,8 \pm 13,6 \mathrm{mg} \mathrm{dL}^{-1}$. Nos animais do grupo 1, observou-se comportamento cúbico para esse parâmetro; os maiores valores foram verificados na dieta 5, e os menores, na dieta 4(Figura 3). Embora não tenha havido diferença entre as dietas, no grupo 2, os valores absolutos de lactato aumentaram gradativamente à medida que as concentrações de fósforo na dieta se elevaram. Ludtke et al. (2010) utilizaram o lactato plasmático como marcador de estresse em suínos e relataram maiores concentrações em animais expostos ao calor. Durante o estresse por calor, ocorre maior liberação de cortisol e adrenalina, que, por sua vez, produzem maior quantidade de ácido láctico, resultante da degradação do glicogênio muscular, o que eleva suas concentrações plasmáticas. Além disso, animais em ambientes quentes podem ter suas concentrações de lactato plasmático aumentadas pelo aumento de captação de oxigênio pelas hemácias, em razão da taquipneia observada em animais nesses ambientes. Isso aumenta o metabolismo das hemácias, o que aumenta a produção de lactato.

$\mathrm{Na}$ avaliação do TSH e do $\mathrm{T}_{4}$ livre, os valores encontrados antes dos tratamentos foram de $0,1 \pm 0,2 \mathrm{uIU} \mathrm{L} \mathrm{L}^{-1}$ e $0,7 \pm 0,2 \mathrm{ng} \mathrm{dL}^{-1}$, respectivamente. As concentrações séricas de TSH não diferiram entre as dietas, mas diferiram entre os grupos (Tabela 4). Entretanto, não houve interação entre esses valores. Já as concentrações séricas de $\mathrm{T}_{4}$ livre apresentaram comportamento quadrático nos grupos 1 e 2 (Figura 2),

Tabela 3. Concentrações séricas de albumina, proteína total, ureia, creatinina e concentrações plasmáticas de glicose e lactato, em leitões em fase inicial de crescimento (15-30 kg), alimentados com dietas com diferentes suplementações de fósforo disponível e mantidos em ambiente termoneutro (grupo $1,24^{\circ} \mathrm{C}$ ) ou quente (grupo $2,34^{\circ} \mathrm{C}$ ) ${ }^{(1)}$.

\begin{tabular}{|c|c|c|c|c|c|}
\hline \multirow[t]{2}{*}{ Grupo } & \multicolumn{5}{|c|}{ Nível de fósforo disponível nas dietas (\%) } \\
\hline & 0,107 & 0,214 & 0,321 & 0,428 & 0,535 \\
\hline & \multicolumn{5}{|c|}{ Albumina $\left(\mathrm{g} \mathrm{L}^{-1}\right)$} \\
\hline 1 & $38 \pm 2 \mathrm{Aa}$ & $38 \pm 2 \mathrm{Aa}$ & $39 \pm 2 \mathrm{Aa}$ & $38 \pm 2 \mathrm{Aa}$ & $40 \pm 2 \mathrm{Aa}$ \\
\hline \multirow[t]{2}{*}{2} & $37 \pm 3 \mathrm{Aa}$ & $38 \pm 2 \mathrm{Aa}$ & $38 \pm 1 \mathrm{Aa}$ & $38 \pm 1 \mathrm{Aa}$ & $38 \pm 2 \mathrm{Aa}$ \\
\hline & \multicolumn{5}{|c|}{ Proteína total $\left(\mathrm{g} \mathrm{L}^{-1}\right)$} \\
\hline 1 & $58 \pm 2 \mathrm{Aa}$ & $59 \pm 4 \mathrm{Aa}$ & $58 \pm 3 \mathrm{Aa}$ & $57 \pm 2 \mathrm{Aa}$ & $61 \pm 4 \mathrm{Aa}$ \\
\hline \multirow[t]{2}{*}{2} & $58 \pm 3 \mathrm{Aa}$ & $60 \pm 5 \mathrm{Aa}$ & $60 \pm 3 \mathrm{Aa}$ & $58 \pm 4 \mathrm{Aa}$ & $59 \pm 5 \mathrm{Aa}$ \\
\hline & \multicolumn{5}{|c|}{ Ureia $\left(\mathrm{mg} \mathrm{dL}^{-1}\right)$} \\
\hline 1 & $40,2 \pm 6 \mathrm{Aa}$ & $38,9 \pm 5,7 \mathrm{Aa}$ & $43,3 \pm 8,7 \mathrm{Aa}$ & $37,9 \pm 5,3 \mathrm{Aa}$ & $40,7 \pm 10,6 \mathrm{Aa}$ \\
\hline \multirow[t]{2}{*}{2} & $38,9 \pm 9,4 \mathrm{Aa}$ & $39,3 \pm 7 \mathrm{Aa}$ & $40 \pm 9,4 \mathrm{Aa}$ & $36,7 \pm 5,6 \mathrm{Aa}$ & $37,7 \pm 10,3 \mathrm{Aa}$ \\
\hline & \multicolumn{5}{|c|}{ Creatinina $\left(\mathrm{mg} \mathrm{dL}^{-1}\right)$} \\
\hline 1 & $1 \pm 0,8 \mathrm{Aa}$ & $1 \pm 0,8 \mathrm{Ba}$ & $1 \pm 0,1 \mathrm{Aa}$ & $1 \pm 0,9 \mathrm{Aa}$ & $1 \pm 0,7 \mathrm{Aa}$ \\
\hline \multirow[t]{2}{*}{2} & $1,1 \pm 0,3 \mathrm{Aa}$ & $1,2 \pm 0,1 \mathrm{Aa}$ & $1,2 \pm 0,8 \mathrm{Aa}$ & $1,1 \pm 0,3 \mathrm{Aa}$ & $0,9 \pm 0,4 \mathrm{Aa}$ \\
\hline & \multicolumn{5}{|c|}{ Glicose $\left(\mathrm{mg} \mathrm{dL}^{-1}\right)$} \\
\hline 1 & $90,3 \pm 10,9$ Aa & $98,3 \pm 9,5 \mathrm{Aa}$ & $95,7 \pm 14,5 \mathrm{Aa}$ & $93,7 \pm 10,8 \mathrm{Aa}$ & $96,2 \pm 9,9 \mathrm{Aa}$ \\
\hline \multirow[t]{2}{*}{2} & $81,5 \pm 8,8 \mathrm{Ba}$ & $86,5 \pm 5,9 \mathrm{Ba}$ & $86,8 \pm 17,9 \mathrm{Aa}$ & $92,4 \pm 5,6 \mathrm{Aa}$ & $92,8 \pm 7,8 \mathrm{Aa}$ \\
\hline & \multicolumn{5}{|c|}{ Lactato $\left(\mathrm{mg} \mathrm{dL}^{-1}\right)$} \\
\hline 1 & $30,2 \pm 7,1 \mathrm{Abc}$ & $36,5 \pm 10,3 \mathrm{Aabc}$ & $40,1 \pm 11,9 \mathrm{Aab}$ & $27,1 \pm 5,8 \mathrm{Ac}$ & $44,5 \pm 14 \mathrm{Aa}$ \\
\hline 2 & $38 \pm 14,9 \mathrm{Aa}$ & $40,1 \pm 15,7 \mathrm{Aa}$ & $46,4 \pm 18,7 \mathrm{Aa}$ & $44,3 \pm 8,3 \mathrm{Aa}$ & $46,2 \pm 14,6 \mathrm{Aa}$ \\
\hline
\end{tabular}

${ }^{(1)}$ Médias seguidas de letras iguais, minúsculas nas linhas e maiúsculas nas colunas, para uma mesma característica, não diferem pelo teste de Tukey ou Wilcoxon (lactato), a $5 \%$ de probabilidade. 
com maiores valores na dieta 1 , no grupo 1 . No grupo 2, observaram-se resultados semelhantes, exceto para a dieta 2, que não diferiu das demais (Tabela 4). $\mathrm{Na}$ avaliação entre os grupos 1 e 2, as dietas 1 e 3 apresentaram as menores concentrações no grupo 2 . Enquanto as concentrações de TSH permaneceram constantes, com o aumento da suplementação de fósforo, os valores da $\mathrm{T}_{4}$ livre tenderam a diminuir nos animais do grupo 2. Pode-se inferir, portanto, que o calor diminui o estímulo do TSH sobre a tireoide, o que levaria à diminuição na síntese de tiroxina $\left(\mathrm{T}_{4}\right)$, para que o metabolismo basal diminuísse na tentativa de reduzir a produção de calor. Dahlke et al. (2005) e

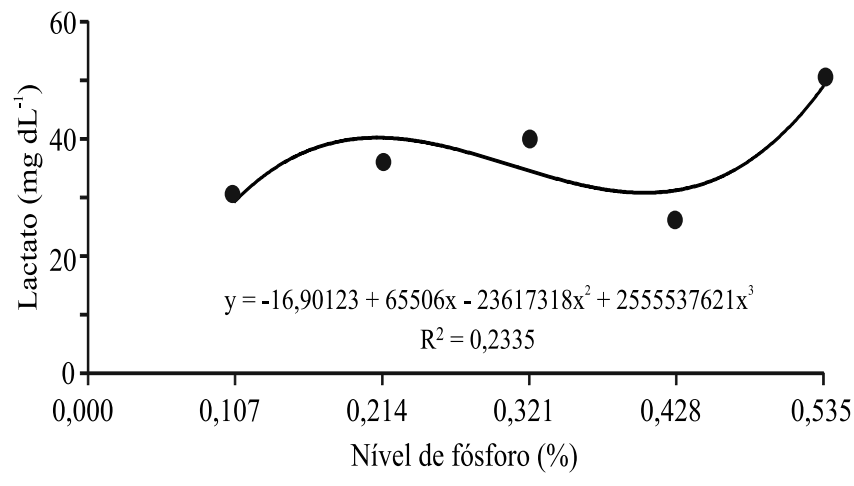

Figura 3. Concentrações plasmáticas de lactato de leitões em fase inicial de crescimento (15-30 kg), no grupo 1, em função de diferentes níveis de fósforo na dieta.
Souza et al. (2012), em estudos com caprinos e frangos em ambientes de alta temperatura, constataram que essas alterações fisiológicas ocorrem como mecanismo compensatório do organismo. Os valores de $\mathrm{T}_{4}$ livre não ultrapassaram os valores mínimos obtidos no início do estudo.

Os valores séricos da fosfatase alcalina total apresentaram comportamento quadrático no grupo $1 \mathrm{e}$ linear no grupo 2 (Figura 2). No grupo 1, as dietas 4 e 5 diferiram da dieta controle; no grupo 1 , no entanto, não houve diferenças entre as dietas. No grupo 2, as dietas 3, 4 e 5 diferiram do controle (Tabela 4). Em relação aos ambientes, as dietas 3 e 5 apresentaram os maiores valores no grupo 2 . Os valores da fosfatase alcalina total estiveram dentro dos limites fisiológicos para a espécie, propostos por Kaneko et al. (2008) e Radostits et al. (2010).

$\mathrm{Na}$ avaliação da fosfatase alcalina óssea, os valores no início da pesquisa foram de $92 \pm 24,2 \mathrm{ng} \mathrm{dL}^{-1}$. No grupo 1, os resultados para essa enzima sofreram influência das dietas, com menores concentrações nos animais que receberam suplementação com fósforo. Entretanto, nos animais do grupo 2, não houve diferença entre as dietas. Também não houve diferenças entre os ambientes estudados. Em ambos os ambientes, notou-se um decréscimo nas concentrações séricas da fosfatase alcalina óssea com a suplementação de fósforo. Esse resultado, possivelmente, deveu-se ao desequilíbrio das relações cálcio:fósforo sanguíneo e ósseo, visto que a maior concentração dessa enzima

Tabela 4. Concentrações séricas de $\mathrm{TSH}, \mathrm{T}_{4}$ livre, fosfatase alcalina total e fosfatase alcalina óssea de leitões em fase inicial de crescimento (15-30 kg), alimentados com dietas com diferentes suplementações de fósforo disponível e mantidos em ambiente termoneutro (grupo $1,24^{\circ} \mathrm{C}$ ) ou quente (grupo $\left.2,34^{\circ} \mathrm{C}\right)^{(1)}$.

\begin{tabular}{|c|c|c|c|c|c|}
\hline \multirow[t]{2}{*}{ Grupo } & \multicolumn{5}{|c|}{ Nível de fósforo disponível nas dietas (\%) } \\
\hline & 0,107 & 0,214 & 0,321 & 0,428 & 0,535 \\
\hline & \multicolumn{5}{|c|}{ TSH (uIU L-1) } \\
\hline 1 & $0,2 \pm 0,4 \mathrm{Ba}$ & $0,2 \pm 0,3 \mathrm{Ba}$ & $0,1 \pm 0,1 \mathrm{Ba}$ & $0,2 \pm 0,3 \mathrm{Aa}$ & $0,2 \pm 0,2 \mathrm{Ba}$ \\
\hline \multirow[t]{2}{*}{2} & $0,3 \pm 0,2 \mathrm{Aa}$ & $0,3 \pm 0,1 \mathrm{Aa}$ & $0,3 \pm 0,2 \mathrm{Aa}$ & $0,2 \pm 0,6 \mathrm{Aa}$ & $0,3 \pm 0,1 \mathrm{Aa}$ \\
\hline & \multicolumn{5}{|c|}{$\mathrm{T}_{4}$ livre $\left(\mathrm{ng} \mathrm{dL}^{-1}\right)$} \\
\hline 1 & $1 \pm 0,2 \mathrm{Aa}$ & $0,8 \pm 0,1 \mathrm{Ab}$ & $0,7 \pm 0,2 \mathrm{Ab}$ & $0,6 \pm 0,7 \mathrm{Ab}$ & $0,67 \pm 0,1 \mathrm{Ab}$ \\
\hline \multirow[t]{2}{*}{2} & $0,8 \pm 0,2 \mathrm{Ba}$ & $0,7 \pm 0,1 \mathrm{Aab}$ & $0,6 \pm 0,8 \mathrm{Bb}$ & $0,6 \pm 1 \mathrm{Ab}$ & $0,6 \pm 0,1 \mathrm{Ab}$ \\
\hline & \multicolumn{5}{|c|}{ Fosfatase alcalina total $\left(\mathrm{U} \mathrm{L}^{-1}\right)$} \\
\hline 1 & $285,7 \pm 92,4 \mathrm{Aa}$ & $212,8 \pm 55,8 \mathrm{Aac}$ & $191,6 \pm 20,6$ Aac & $169,5 \pm 25,1 \mathrm{Abc}$ & $174 \pm 56,1 \mathrm{Abc}$ \\
\hline \multirow[t]{2}{*}{2} & $212,6 \pm 65,8 \mathrm{Aa}$ & $169,1 \pm 53,3 \mathrm{Aab}$ & $140,4 \pm 34,1 \mathrm{Bb}$ & $154,3 \pm 28 \mathrm{Ab}$ & $131,5 \pm 31,6 \mathrm{Bb}$ \\
\hline & \multicolumn{5}{|c|}{ Fosfatase alcalina óssea $\left(\mathrm{ng} \mathrm{dL}^{-1}\right)$} \\
\hline 1 & $95,7 \pm 29,3 \mathrm{Aa}$ & $64,1 \pm 23,7 \mathrm{Ab}$ & $55,7 \pm 14,9 \mathrm{Ab}$ & $48,9 \pm 14,2 \mathrm{Ab}$ & $48,5 \pm 20,9 \mathrm{Ab}$ \\
\hline 2 & $75,4 \pm 30,0 \mathrm{Aa}$ & $52,6 \pm 25,6 \mathrm{Aa}$ & $44,2 \pm 11,4 \mathrm{Aa}$ & $50,2 \pm 11,6 \mathrm{Aa}$ & $43,1 \pm 11,6 \mathrm{Aa}$ \\
\hline
\end{tabular}

${ }^{(1)}$ Médias seguidas de letras iguais, minúsculas nas linhas e maiúsculas nas colunas, para uma mesma característica, não diferem pelo teste de Tukey ou Wilcoxon (fosfatase alcalina total), a $5 \%$ de probabilidade. 
está presente nos osteoblastos para a produção da matriz óssea (Klein, 2014). A fosfatase alcalina óssea é considerada como um marcador de atividade óssea em cães (Sousa et al., 2011) e em humanos (Cremers et al., 2008).

\section{Conclusões}

1. Parâmetros bioquímicos sanguíneos retratam o estado metabólico de suínos e podem ser usados para avaliação do balanço nutricional e para detecção precoce de alterações metabólicas.

2. O aumento nos teores de fósforo na dieta de suínos aumenta o ganho de peso corporal, independentemente do ambiente estudado.

3. O aumento dos teores de fósforo na dieta de suínos causa desequilíbrio das relações cálcio:fósforo sanguíneos.

4. O estresse por calor diminui as concentrações de glicose, fosfatase alcalina total e $\mathrm{T}_{4}$ livre, e aumenta as de lactato.

\section{Agradecimentos}

À Coordenação de Aperfeiçoamento de pessoal de Nível Superior (Capes), pela concessão de bolsa; e à Fundação de Amparo à Pesquisa do Estado de Minas Gerais (Fapemig), pelo apoio financeiro.

\section{Referências}

ALEBRANTE, L.; DONZELE, J.L.; OLIVEIRA, R.F.M. de; SARAIVA, A.; GUIMARÃES, S.E.F.; FERREIRA, A.S. Available phosphorus levels in diets for pigs with high genetic potential for lean meat deposition kept in thermoneutral environment from 15 to $30 \mathrm{~kg}$. Revista Brasileira de Zootecnia, v.40, p.323-330, $2011 \mathrm{a}$. DOI: $10.1590 / \mathrm{S} 1516-35982011000200013$.

ALEBRANTE, L.; DONZELE, J.L.; OLIVEIRA, R.F.M. de; SARAIVA, A.; GUIMARÃES, S.E.F; FERREIRA, A.S.; SILVA, F.C. de O.; ABREU, M.L.T. de. Available phosphorus for 15to $30-\mathrm{kg}$ pigs kept in hot environment. Revista Brasileira de Zootecnia, v.40, p.2725-2731, 2011b. DOI: 10.1590/ S1516-35982011001200016.

BRÊTAS, A. de A.; FERREIRA, R.A.; AMARANTE JÚNIOR, V. da S.; PEREIRA, W.E.; FONSECA, J.B.; CALDAS, F.R.L. Balanço eletrolítico para suínos machos castrados em crescimento mantidos em ambiente de alta temperatura. Ciência e Agrotecnologia, v.35, p.186-194, 2011. DOI: 10.1590/S1413-70542011000100024.

BUFFINGTON, D.E.; COLlAZO-AROCHO, A.; CANTON, G.H. Black globe- humidity index (BGHI) as comfort equation for dairy cows. Transactions of the ASAE, v.24, p.711-714, 1981. DOI: $10.13031 / 2013.34325$.
CAMPOS, J.A.; TINÔCO, I. de F.F.; BAÊTA, F. da C.; SILVA, J.N. da; CARVALHO, C.S.; MAUIRI, A.L. Ambiente térmico e desempenho de suínos em dois modelos de maternidade e creche. Revista Ceres, v.55, p.187-193, 2008.

COFFEY, R.D.; PARKER, G.R.; LAURENT, K.M. Feeding growing-finishing pigs to maximize lean grow rate. Lexington: University of Kentucky, 2000. Available at: <http://www. animalgenome.org/edu/PIH/prod_grow_finish.pdf $>$. Accessed on: 4 May 2013.

CONSTABLE, P.D. Hyperchloremic acidosis: the classic example of strong ion acidosis. Anesthesia and Analgesia, v.96, p.919-922, 2003. DOI: 10.1213/01.ANE.0000053256.77500.9D.

CREMERS, S.; GARNERO, P.; SEIBEL, M.J. Biochemical markers of bone metabolism. In: BILEZIKIAN, J.P.; RAISZ, L.G.; JOHN MARTIN, T. Principles of bone biology. $3^{\text {rd }}$ ed. San Diego: Academic Press, 2008. p.1857-1881. DOI: 10.1016/ B978-0-12-373884-4.00020-3.

DAHLKE, F.; GONZALES, E.; FURLAN, R.L.; GADELHA, A.; MAIORKA, A.; FARIA FILHO, D.E.; ROSA, P.S. Efeito da temperatura ambiente sobre hormônios tireoideanos, temperatura corporal e empenamento de frangos de corte, fêmeas, de diferentes genótipos. Acta Scientiarum. Animal Sciences, v.27, p.391-397, 2005.

DANTAS, W. de M.F.; RIBEIRO FILHO, J.D.; GUIMARÃES, J.D.; FARIAS, S.K; GUIMARÃES, S.E.F.; SARAIVA, A.; OLIVEIRA, T.T. de. Perfil eletrolítico e peso corporal em suínos submetidos a dietas com diferentes teores de fósforo. Pesquisa Agropecuária Brasileira, v.45, p.1205-1210, 2010. DOI: 10.1590/ S0100-204X2010001000021.

DÍAZ GONZÁLEZ, F.H.; SILVA, S.C. da. Introdução à bioquímica clínica veterinária. 2.ed. Porto Alegre: UFRGS, 2006. 360p.

KANEKO, J.J.; HARVEY, J.W.; BRUSS, M.L. Clinical biochemistry of domestic animals. $5^{\text {th }}$ ed. San Diego: Academic Press, 2008. 932p.

KIEFER, C.; SANCHES, J.F.; SILVA, A.P.; YOSHIDA, F.Y.; SILVA, C.M. Sódio e balanço eletrolítico em dietas para leitões dos 8 aos $25 \mathrm{~kg}$ mantidos em ambiente de alta temperatura. Ciência Animal Brasileira, v.11, p.503-508, 2010. DOI: 10.5216/cab. v11i3.5156.

KLEIN, B.G. Cunningham: tratado de fisiologia veterinária. 5.ed. Rio de Janeiro: Elsevier, 2014. 624p.

LUDTKE， C.B.; SILVEIRA, E.T.F.; BERTOLONI, W.; ANDRADE, J.C. de; BUZELLI, M.L.T.; BRESSA, L.R.; SOARES, G.J.D. Bem-estar e qualidade de carne de suínos submetidos a diferentes técnicas de manejo pré-abate. Revista Brasileira de Saúde e Produção Animal, v.11, p.231-241, 2010.

MANNO, M.C.; OLIVEIRA, R.F.M. de; DONZELE, J.L.; FERREIRA, A.S.; OLIVEIRA, W.P. de; LIMA, K.R. de S.; VAZ, R.G.M.V. Efeito da temperatura ambiente sobre o desempenho de suínos dos 15 aos $30 \mathrm{~kg}$. Revista Brasileira de Zootecnia, v.34, p.1963-1970, 2005. DOI: 10.1590/S1516-35982005000600021.

RADOSTITS, O.M.; GAY, C.C.; HINCHCLIFF, K.W.; CONSTABLE, P.D. Veterinary medicine. $10^{\text {th }}$ ed. Philadelphia: Elsevier, 2010. 2162p.

Pesq. agropec. bras., Brasília, v.49, n.11, p.901-910, nov. 2014 DOI: 10.1590/S0100-204X2014001100009 
ROSTAGNO, H.S.; ALBINO, L.F.T.; DONZELE, J.L.; GOMES, P.C.; OLIVEIRA, R.F.; LOPES, D.C.; FERREIRA, A.S.; BARRETO, S.L.T. Tabelas brasileiras para aves e suínos: composição de alimentos e exigências nutricionais. 2.ed. Viçosa: Ed. da UFV, 2005. 186p.

SAEG: sistema para análises estatísticas. Versão 9.1. Viçosa: Ed. da UFV, 2007. 149p.

SARAIVA, A.; DONZELE, J.L.; OLIVEIRA, R.F.M. de; ABREU, M.L.T.; SILVA, F.C. de O.; SANTOS, F. de A. Available phosphorus levels in diets for swine from 15 to $30 \mathrm{~kg}$ genetically selected for meat deposition. Revista Brasileira de Zootecnia, v.38, p.307-313, 2009. DOI: 10.1590/S1516-35982009000200013.

SAS INSTITUTE. SAS user's guide. Version 9.0. Cary: SAS Institute, 2000. 544p.

SOUSA, C.; ABREU, H.; VIEGAS, C.; AZEVEDO, J.; REIS, R.; GOMES, M.; DIAS, I. Serum total and bone alkaline phosphatase and tartrate-resistant acid phosphatase activities for the assessment of bone fracture healing in dogs. Arquivo Brasileiro de Medicina Veterinária e Zootecnia, v.63, p.1007-1011, 2011. DOI: 10.1590/ S0102-09352011000400029.

SOUZA, P.T. de; SALLES, M.G.F.; ARAÚJO, A.A. de. Impacto do estresse térmico sobre a fisiologia, reprodução e produção de caprinos. Ciência Rural, v.42, p.1888-1895, 2012. DOI: 10.1590/ S0103-84782012005000072.

TALAMINI, D.J.D.;MARTINS, F.M.; ARBOIT, C.; WOLOZSIN, N. Custos agregados da produção integrada de suínos nas fases de leitões e de terminação. Revista Custos e Agronegócios On Line. v.2, p.64-83, 2006. Edição especial.

TEIXEIRA, A. de O.; LOPES, D.C.; LOPES, J.B.; VITTI, D.M.S.S.; GOMES, P.C.; ROSTAGNO, H.S.; MOREIRA, J.A.; INÁCIO, F. Determinação da biodisponibilidade de fósforo de diferentes fontes pela técnica de diluição isotópica, em suínos em crescimento. Revista Brasileira de Zootecnia, v.33, p.1231-1237, 2004. DOI: 10.1590/S1516-35982004000500015.

Recebido em 13 de maio de 2014 e aprovado em 28 de outubro de 2014 